ARTICLE

DOI: $10.1038 / \mathrm{s} 41467-017-00186-3$

\title{
Organocatalyzed synthesis of fluorinated poly(aryl thioethers)
}

Nathaniel H. Park ${ }^{1}$, Gabriel dos Passos Gomes (1) ${ }^{2}$, Mareva Fevre, Gavin O. Jones ${ }^{1}$, Igor V. Alabugin ${ }^{2}$ \& James L. Hedrick ${ }^{1}$

The preparation of high-performance fluorinated poly(aryl thioethers) has received little attention compared to the corresponding poly(aryl ethers), despite the excellent physical properties displayed by many polysulfides. Herein, we report a highly efficient route to fluorinated poly(aryl thioethers) via an organocatalyzed nucleophilic aromatic substitution of silyl-protected dithiols. This approach requires low catalyst loadings, proceeds rapidly at room temperature, and is effective for many different perfluorinated or highly activated aryl monomers. Computational investigations of the reaction mechanism reveal an unexpected, concerted $\mathrm{S}_{\mathrm{N}} \mathrm{Ar}$ mechanism, with the organocatalyst playing a critical, dual-activation role in facilitating the process. Not only does this remarkable reactivity enable rapid access to fluorinated poly(aryl thioethers), but also opens new avenues for the processing, fabrication, and functionalization of fluorinated materials with easy removal of the volatile catalyst and TMSF byproducts.

\footnotetext{
${ }^{1}$ IBM Almaden Research Center, 650 Harry Road, San Jose, CA 95120, USA. ${ }^{2}$ Department of Chemistry and Biochemistry, Florida State University, Tallahassee, FL 32310, USA. Correspondence and requests for materials should be addressed to J.L.H. (email: hedrick@us.ibm.com)
} 
F luorinated materials exhibit many highly desirable properties such as increased chemical resistance, hydrophobicity, and thermal stability. One common route to introduce fluorine into polymer backbones is through the polycondensation of perfluoroarene-containing monomers (Fig. 1) ${ }^{1-12}$. These perfluorinated aromatic polymers impart increased order and stability to processed materials by forming energetically favorable $\pi-\pi$ stacking arrangements with non-perfluoroarenes ${ }^{13,14}$, as evidenced by their thermal properties ${ }^{4}$. Fluorinated aromatic polymers often possess a lower refractive index and optical loss than non-fluorinated analogs, making them excellent candidate materials for optical material applications ${ }^{3,5}$. Given the advantages of having perfluoroarene units in polymers, we felt that the preparation of high performance fluorinated poly(aryl thioethers) would be ideally suited for use in coating and device applications.

As with perfluoroaryl-containing polymers, poly(aryl thioethers) are attractive materials that display excellent performance characteristics such as high thermal stability and hydrophobicity ${ }^{15}$. Poly(aryl thioethers) are traditionally prepared via nucleophilic aromatic substitution $\left(\mathrm{S}_{\mathrm{N}} \mathrm{Ar}\right)$ under conditions that utilize stoichiometric amounts of base, extended reaction times, or in some cases, high temperatures ${ }^{16-21}$. Although these conditions have been utilized for the incorporation of perfluoroaryl-containing monomers into poly(aryl thioethers $)^{22}$, they are not ideal, given the propensity of perfluoroarenes to undergo multiple substitutions $3,23-26$ potentially leading to uncontrolled branching or cross-linking. Additionally, the stoichiometric amount of salt generated under standard $\mathrm{S}_{\mathrm{N}} \mathrm{Ar}$ conditions could hamper further applications such as casting films to prepare hydrophobic surfaces. To circumvent the necessity of using stoichiometric base for the $S_{N} A r$ reaction, we sought conditions for catalytic generation and reaction of thiolates. Here, we focused our attention on trimethylsilyl-protected thioethers, which could be cleaved by a catalyst to reveal a thiolate nucleophile. This intermediate could then undergo $S_{N} A r$ with a perfluoroarene, thereby liberating trimethylsilylfluoride (TMSF) and regenerating the catalyst for subsequent reactions. Repetition of this cycle would give rise to the desired fluorinated poly(aryl thioether). Although similar protocols have been utilized for the preparation of polyethers ${ }^{27,28}$, polysulfoxides ${ }^{29}$, polythiophenes ${ }^{1,5}$, and poly(phenyleneethynylene) $s^{3}$, there are no reports on the use of silylated dithiols as monomers. Thus, in order to take full advantage of the unique properties of fluoropolymers and poly (aryl thioethers), we developed a catalytic approach for the direct polymerization of perfluoroarenes into poly(aryl thioether) systems under mild conditions. This approach facilitates the polymerization of a variety of thiol nucleophiles and fluoroarenes and concurrent computational investigation of the reaction mechanism reveals a unique role of the catalyst in facilitating the polymerization process.

\section{Results}

Evaluation of polymerization catalysts. We began by preparing thioether 1a (Table 1) as the trimethylsilane (TMS) protected nucleophile. By reacting 1a with hexafluorobenzene using $5 \mathrm{~mol} \%$ DBU as the organocatalyst ${ }^{27}$, a swift exotherm was observed, coupled with the formation of fluorotrimethylsilane (TMSF) and a rapid precipitation of polymeric material having a $M_{\mathrm{n}}$ of $8456 \mathrm{~g} / \mathrm{mol}$ and a dispersity of 4.88 (entry 1 , Table 1 ). The fast rate of reaction was consistent with preliminary time course experiments at higher catalyst loadings, which revealed the complete consumption of hexafluorobenzene within several seconds (see Supplementary Fig. 1). For further comparison of different catalyst systems and catalyst loadings, we selected $15 \mathrm{~min}$ as a benchmark reaction time. The large dispersity observed (entry 1, Table 1) may be the result of cross-linking or branching via multiple substitutions on the arene ring and would be consistent with the high reactivity of hexafluorobenzene $e^{3,23-26}$ However, examination of the ${ }^{19} \mathrm{~F}$ NMR spectrum reveals a clean singlet indicative of a symmetrically substituted perfluoroarene ring and therefore the dispersity of the isolated material is more likely a result of kinetic quenching from the precipitation of the polymeric material. By lowering the catalyst loading to 1 or 0.5 mol \%, the corresponding $M_{\mathrm{n}}$ and dispersity of the polymers decreased while still affording short reaction times (entries 2 and 3 , Table 1). Performing the reaction inside a glovebox under strictly anhydrous conditions afforded a higher $M_{\mathrm{n}}$ and dispersity, indicating that the polymerization reaction is likely sensitive to water and ambient moisture (entry 4, Table 1).

Other catalyst systems in addition to DBU were also evaluated. Guanidine containing catalysts such as TBD and DMC, gave similar results as compared to DBU (entries 5 and 6, Table 1). TBD afforded a higher dispersity, presumably due to increased basicity and hence reactivity. Less basic catalysts, such as triethylamine, DIEA, and DABCO, all gave polymers with a narrower dispersity than their more strongly basic counterparts (entries 8-10, Table 1). However, the corresponding molecular weights of the resultant polymers were lower and longer reaction times were required. By heating the reaction with $10 \mathrm{~mol} \%$ of diisopropylethylamine; however, results similar to those using DBU could be obtained (entry 11, Table 1).

Thermal properties of fluorinated poly(aryl thioethers). The thermal properties $\mathbf{1 b}$ were investigated by thermogravimetric analysis (TGA), differential scanning calorimetry (DSC) and dynamic mechanical analysis (DMA). The $T_{2 \%}$ of $\mathbf{1 b}$, which was isolated by precipitation prior to analysis, was found at $333^{\circ} \mathrm{C}$ (see Supplementary Fig. 2). Figure 1 shows the reverse Cp plot of the same isolated polymer (Fig. 1b), as well as DMA traces of in situ polymerized solutions of $1 \mathbf{a}$ and $\mathrm{C}_{6} \mathrm{~F}_{6}$ on a support braid (Fig. 1a) in NMP. Two thermal transitions were detected on the DSC thermogram in the studied temperature range: a glass transition around $-18^{\circ} \mathrm{C}$ and a melting endotherm around $105^{\circ} \mathrm{C}$, evidence of the semi-crystalline morphology of $\mathbf{1 b}$ (Fig. 1b), consistent with other perfluoroarene-containing poly(aryl

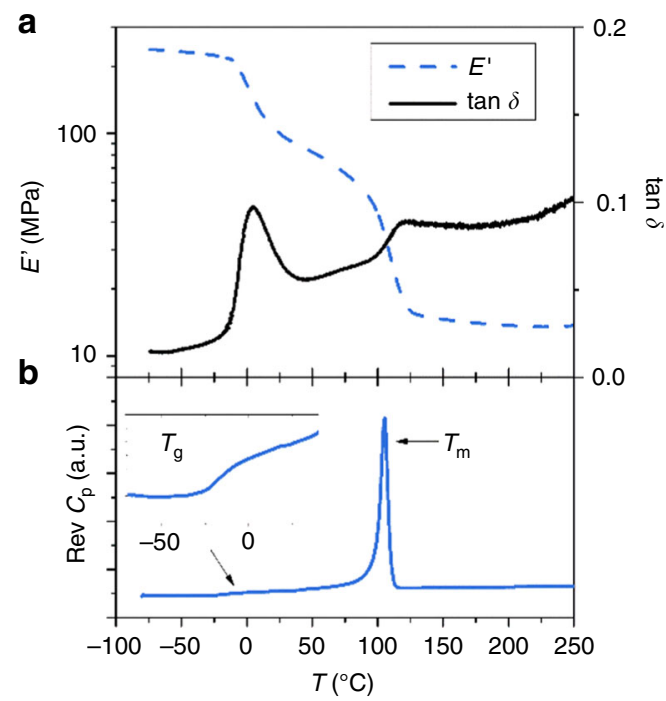

Fig. 1 Thermal analysis of fluorinated poly(aryl thioethers). a DMA analysis of $\mathbf{1 b}$. $\mathbf{b}$ DSC analysis of $\mathbf{1 b}$. The sample used for DSC analysis was isolated by precipitation, while a braid for DMA was prepared with NMP-solutions of $\mathbf{1 a}$ and hexafluorobenzene 


\section{Table 1 Evaluation of catalysts and catalyst loadings}
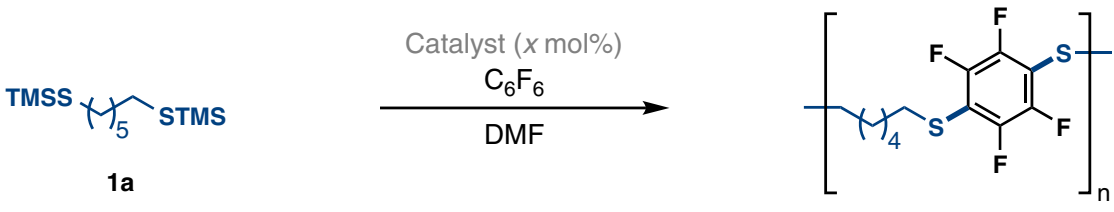

Aryl polythioether (1b)

\begin{tabular}{|c|c|c|c|c|c|c|}
\hline Entry & Catalyst & Mol \% & Time & $M_{n}^{\mathrm{a}}$ & $M_{w}^{\mathrm{a}}$ & $\oplus^{a}$ \\
\hline 1 & DBU & 5 & $15 \mathrm{~min}$ & 8456 & 41241 & 4.88 \\
\hline 2 & DBU & 1 & $15 \mathrm{~min}$ & 7128 & 22597 & 3.17 \\
\hline 3 & DBU & 0.5 & $15 \mathrm{~min}$ & 6926 & 17438 & 2.52 \\
\hline $4^{b}$ & DBU & 0.5 & $15 \mathrm{~min}$ & 33210 & 120743 & 3.64 \\
\hline 5 & TBD & 0.5 & $15 \mathrm{~min}$ & 6017 & 24047 & 4.00 \\
\hline 6 & DMC & 0.5 & $15 \mathrm{~min}$ & 6804 & 19075 & 2.80 \\
\hline 7 & TBAF & 0.5 & $15 \mathrm{~min}$ & 7509 & 44617 & 5.94 \\
\hline $8^{c}$ & $\mathrm{Et}_{3} \mathrm{~N}$ & 10 & $14 \mathrm{~h}$ & 2455 & 4159 & 1.69 \\
\hline $9^{d}$ & $\mathrm{PPr}_{2} \mathrm{NEt}$ & 10 & $14 \mathrm{~h}$ & 3043 & 5844 & 1.92 \\
\hline $10^{\mathrm{e}}$ & $\mathrm{DABCO}$ & 10 & $14 \mathrm{~h}$ & 2750 & 4704 & 1.71 \\
\hline $11^{f}$ & $i \mathrm{Pr}_{2} \mathrm{NEt}$ & 10 & $16 \mathrm{~h}$ & 9267 & 22868 & 2.47 \\
\hline
\end{tabular}

Reagents and conditions: 1a (0.25-0.5 mmol), hexafluorobenzene (0.25-0.5 mmol), catalyst (0.5-10 mol \%), DMF (1 M), rt, 15 min-16 h. DBU: 1,8-diazobicyclo(5.4.0)undec-7-ene, TBD: triazabicyclodecene, DMC: $N, N^{\prime}$-dicyclohexyl-4-morpholineformamidine, TBAF: tetra-n-butylammonium fluoride, DABCO: 1,4-diazabicyclo(2.2.2)octane

Determined by SEC calibrated with polystyrene standards and using THF as the eluent

beaction run inside glovebox

${ }^{\mathrm{C}}$ Reaction gave $94 \%$ conversion of hexafluorobenzene based on ${ }^{19} \mathrm{~F}$ analysis of crude reaction mixture using $\mathrm{PhCF}_{3}$ as an internal standarc

d $96 \%$ conversion of hexafluorobenzene

e $98 \%$ conversion of hexafluorobenzene

${ }^{\mathrm{f}}$ Reaction run at $100^{\circ} \mathrm{C}$

thioethers $)^{10,22}$. These data were in agreement with the DMA plots, where two E' drops along with two $\tan \delta$ maxima were observed (Fig. 1a).

Evaluation of other monomers. Having investigated the thermal properties of 1a and identified the appropriate catalyst systems and reaction parameters, we next utilized this approach to polymerize other fluoroarene electrophiles and silyl thioethers. Decafluorobiphenyl proved to be an excellent substrate for this reaction and readily polymerized when $\mathbf{1 a}$ was used as the nucleophile with either DBU or TBD as the catalyst ( $\mathbf{2 b}$, entries 1 and 2, Table 2). The TMS thioether of 4,4'-thiodibenzenethiol (2a, Table 2) was also a viable monomer for polymerization with perfluoroarenes. Interestingly, when decafluorobiphenyl was utilized as a co-monomer with $\mathbf{2 a}$, no catalyst was necessary as dissolution of both monomers in DMF was sufficient to induce rapid polymerization to afford $2 \mathrm{c}$ (entry 3 , Table 2 ). Presumably, this reactivity is due to the increased lability of TMS-protected aryl thioethers relative to TMS-protected alkyl thioethers. Both reactions to produce $\mathbf{2 a}$ and $\mathbf{2} \mathbf{b}$ (entries $1-3$, Table 2) gave polymers that are comparable to those produced using unprotected thiols and stoichiometric base (see Supplementary Figs. 3 and 4), highlighting the efficacy of this approach to produce fluorinated poly(aryl thioethers) without stoichiometric salt byproducts. For synthesizing $\mathbf{2 d}$, a catalyst was still needed to facilitate the polymerization of $\mathbf{2} \mathbf{a}$ and hexafluorobenzene (entry 4, Table 2). Non-perfluorinated, yet highly activated aryl electrophiles such as bis(4-fluoro-3-nitrophenyl) sulfone could also be rapidly polymerized under the reaction conditions to form $2 \mathrm{e}$ (Table 2). Unfortunately, the very limited solubility of polymers 2d and 2e prevented their analysis via GPC or NMR, although the $T_{\mathrm{g}}$ for these materials could be obtained from DSC analysis (entries 4 and 5, Table 2).
Computational mechanistic investigation. To understand the origins of the aforementioned remarkable reactivity, we performed computational investigations with the M06-2X density functional method on the mechanisms and energetics for the TBD-catalyzed reaction of hexafluorobenzene with TMSprotected methanethiol (TMS-SMe) (Fig. 2). The nucleophilic attack of TBD on the TMS group of TMS-SMe and displacement of methanethiolate $\left(\mathrm{MeS}^{-}\right)$from the silyl protecting group was identified as a key starting point. This process results in the formation of complex INT1 - where $\mathrm{MeS}^{-}$is hydrogen-bonded to the TBD-TMS cation - and then associates with $\mathrm{C}_{6} \mathrm{~F}_{6}$ to form the productive trimolecular complex INT2 (Fig. 2). The free energy of INT2 formation illustrates that strong enthalpic contributions of attractive supramolecular interactions largely compensate for the unfavorable entropy $(\Delta \mathrm{H}=-8.4 \mathrm{kcal} / \mathrm{mol}, \Delta G=$ $+4.5 \mathrm{kcal} / \mathrm{mol}$ relative to the separated components). Next, complex INT2 reacts to afford the mono-substituted product (Prod1) via TS2, in which TBD promotes the attack of $\mathrm{MeS}^{-}$on the aromatic ring as $\mathrm{F}^{-}$leaves (Fig. 2). This contrasts directly with the typical, stepwise addition-elimination mechanism of $S_{N} A r$ reactions, as the TBD-catalyzed reaction proceeds in a concerted manner where formation of the $\mathrm{C}-\mathrm{S}$ bond is coordinated with scission of the $\mathrm{C}-\mathrm{F}$ bond ${ }^{27,30-32}$. The TBD catalyst serves dual roles in the $\mathrm{S}_{\mathrm{N}} \mathrm{Ar}$ process occurring in TS2: it delivers the $\mathrm{MeS}^{-}$ nucleophile and assists the concomitant displacement of the fluorine atom through a hydrogen bonding interaction (Fig. 2). The free energy barrier for the TBD-assisted thioetherification and fluoride displacement is only $17.6 \mathrm{kcal} / \mathrm{mol}$, presumably owing to synergistic interactions present in the TS, which allows for the scission of a very strong $\mathrm{C}-\mathrm{F}$ bond to proceed with such a small penalty.

These synergistic interactions include the covalent attachment of TBD to the TMS group, which renders the TBD catalyst cationic, and thereby enhances the hydrogen-bond interaction 


\section{Table 2 Evaluation of other monomers for the preparation of poly(aryl thioethers)}

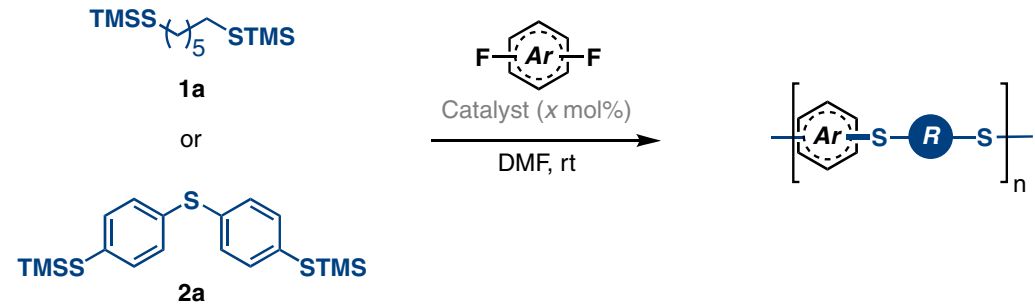

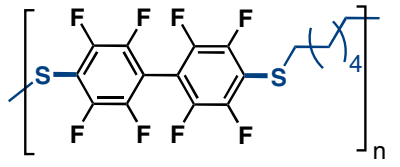

2b

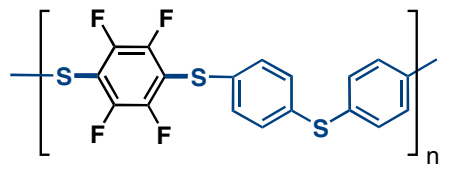

2d<smiles>Cc1ccc(Sc2ccc(Sc3c(F)c(F)c(-c4c(F)c(F)c(S(C)(C)C)c(F)c4F)c(F)c3F)cc2)cc1</smiles>

$2 c$

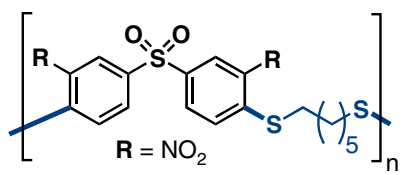

$2 \mathrm{e}$

\begin{tabular}{|c|c|c|c|c|c|c|c|c|}
\hline Entry & Poly. & Cat. & Mol \% & $M_{n}^{\mathrm{a}}$ & $M_{w}^{\mathrm{a}}$ & $\oplus^{a}$ & $\mathrm{~T}_{\mathrm{g}}\left({ }^{\circ} \mathrm{C}\right)^{\mathrm{b}}$ & Yield $^{c}$ \\
\hline$\overline{1^{d}}$ & $2 b$ & TBD & 1 & 9585 & 27898 & 2.91 & - & $94 \%$ \\
\hline $2^{e}$ & $2 b$ & DBU & 1 & 15701 & 40353 & 2.57 & 16.7 & - \\
\hline $3^{d, f}$ & $2 c$ & None & - & 7768 & 47026 & 6.05 & 150.0 & $97 \%$ \\
\hline $4^{f, g}$ & 2d & DBU & 0.25 & - & - & - & 48.0 & $75 \%$ \\
\hline $5^{g}$ & $2 e$ & DBU & 0.5 & - & - & - & 28.0 & $64 \%$ \\
\hline
\end{tabular}

Reagents and conditions: 1a or $\mathbf{2 a}(0.25-0.5 \mathrm{mmol})$, aryl electrophile $(0.25-0.5 \mathrm{mmol})$, catalyst $(0.25-1 \mathrm{~mol} \%), \mathrm{DMF}(1 \mathrm{M}), \mathrm{rt}, 5-15 \mathrm{~min}$

a Determined by SEC using polystyrene standards and THF as the eluent

betermined by DSC on the second heating cycle

"Based on mass of recovered material

dReaction time was $5 \mathrm{~min}$

Reaction time was $15 \mathrm{~min}$

${ }^{f}$ Reaction run inside glovebox

geaction time was $10 \mathrm{~min}$

between the $\mathrm{N}-\mathrm{H}$ group and the sulfide by $\mathrm{n}_{\mathrm{S}} \rightarrow \sigma^{*} \mathrm{H}-\mathrm{N}$ donation due to the decreased electron density on the $\mathrm{N}-\mathrm{H}$ proton (Fig. 3a; see Supplementary Fig. 5 for an S-H scan from the $\mathrm{MeS}^{-}$(TBDTMS) ${ }^{+}$complex). Furthermore, the relatively acidic $\alpha-\mathrm{CH}$ bonds adjacent to the acceptor $\mathrm{N}-\mathrm{H}$ moiety can provide stabilization to the departing fluoride via $\mathrm{C}-\mathrm{H} \bullet \bullet \bullet \mathrm{F}$ interactions (see Supplementary Figs. 6 and 7 for computed natural charges). Electrostatic stabilization due to such interactions has been reported to be significant, even in the absence of strong $\mathrm{n}_{\mathrm{F}} / \sigma^{*} \mathrm{C}-\mathrm{H}$ overlap ${ }^{32}$. Finally, the rigid nature of the catalyst leads to a unique stereoelectronic advantage, as the $\mathrm{N}-\mathrm{H} \bullet \bullet \bullet S$ interaction is associated with the in-plane lone pairs of sulfur, leaving the out-of-plane p-type lone pair fully available for the nucleophilic attack at the aromatic $\pi$-system (Fig. 3a).

The TBD catalyst is regenerated in INT3 by attack of the hydrogen-bonded fluoride anion on the neighboring TMS group in a low-barrier process with a free energy barrier of $6.0 \mathrm{kcal} / \mathrm{mol}$ with reference to the low-lying Prod1 (Fig. 2). The catalyst reenters the cycle by activating a protected thiol to form INT4 in a mechanism analogous to INT1 formation. Complexation of INT4 with $\mathrm{MeSPhF}_{5}$ results in the formation of INT5, a complex similar to INT2, in which the deprotected thiolate is poised for nucleophilic attack para to the thiol substituent (Fig. 2). The presence of SMe substituent in the aromatic ring lowers the free energy barrier by $2.2 \mathrm{kcal} / \mathrm{mol}$, making the second substitution roughly $\sim 33$ times faster than without the SMe (TS5, Fig. 2; Supplementary Fig. 8). The activating effect of the SMe group originates from an unusual geometry at the aryl thioether junction, with the SMe substituent rotating to the near orthogonality out-of-plane of the aromatic ring. The conformational change converts the aryl thioether from a moderate p-donor into a moderate $\sigma$-acceptor (Fig. 3b) ${ }^{33,34}$. This is shown in Fig. $3 b$, as the $\pi_{\mathrm{CC}} \rightarrow \sigma_{\mathrm{S}-\mathrm{C}}^{*}$ interaction increases from 2.2 to 6.8 $\mathrm{kcal} / \mathrm{mol}(4.6 \mathrm{kcal} / \mathrm{mol}$ difference), delocalizing more of the electronic density in the TS and lowering the overall free energy barrier for TS5 (Fig. 2). Following TS5, the TBD catalyst is regenerated in a similar low-barrier process as before (TS6, Fig. 2) to give the final products TMSF, TBD, and the para-disubstituted fluoroarene (INT6, Fig. 2).

\section{Discussion}

Overall, we have developed an organocatalyzed reaction for the synthesis of fluorinated and non-fluorinated poly(aryl thioethers). As opposed to standard $S_{N} A r$ reactions between thiols and perfluoroarenes, our conditions avoid the generation of stoichiometric salt by-products. This advantage, in combination with short reaction times, room temperature conditions, and low to no catalyst loadings, will enable new routes for processing the prepared fluoropolymers into devices and coatings. Computational 


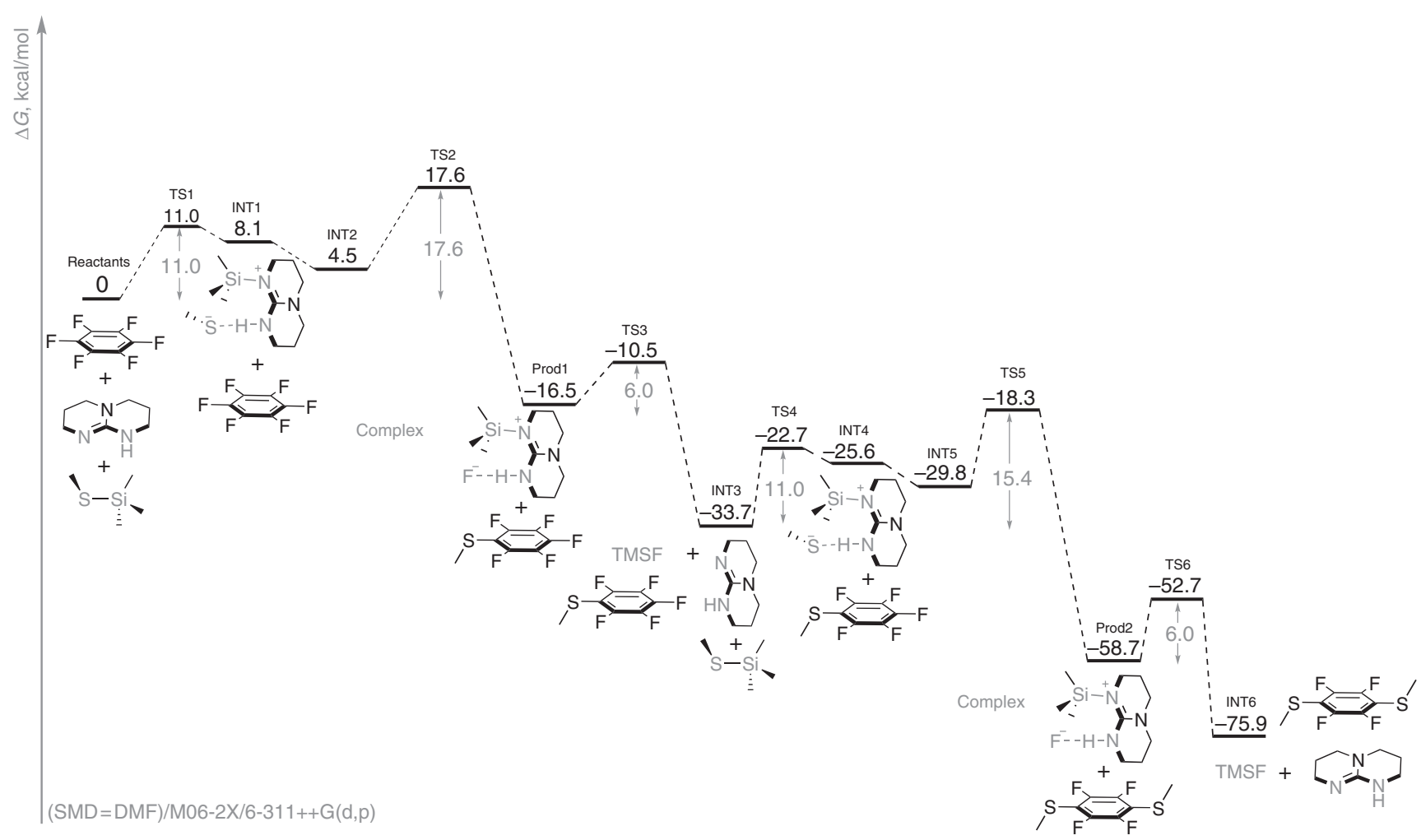

Fig. 2 Computational analysis of the TBD-catalyzed reaction pathway. Free energy profile, structures and energetics for the first two steps in the TBD-catalyzed thioetherification of hexafluorobenzene by MeS-TMS
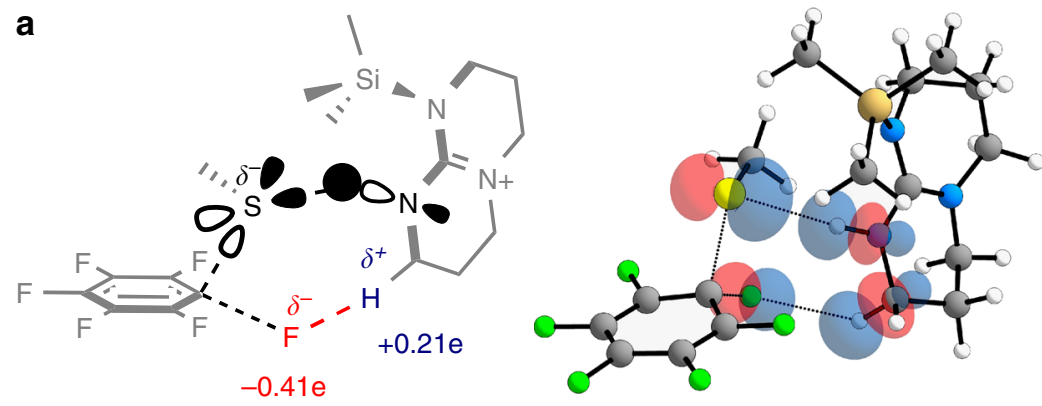

$\mathrm{n}_{\mathrm{S}} \rightarrow \sigma_{\mathrm{H}-\mathrm{N}}^{*}=11.1, \mathrm{n}_{\mathrm{F}} \rightarrow \sigma_{\mathrm{H}-\mathrm{C}}^{*}=0.5$

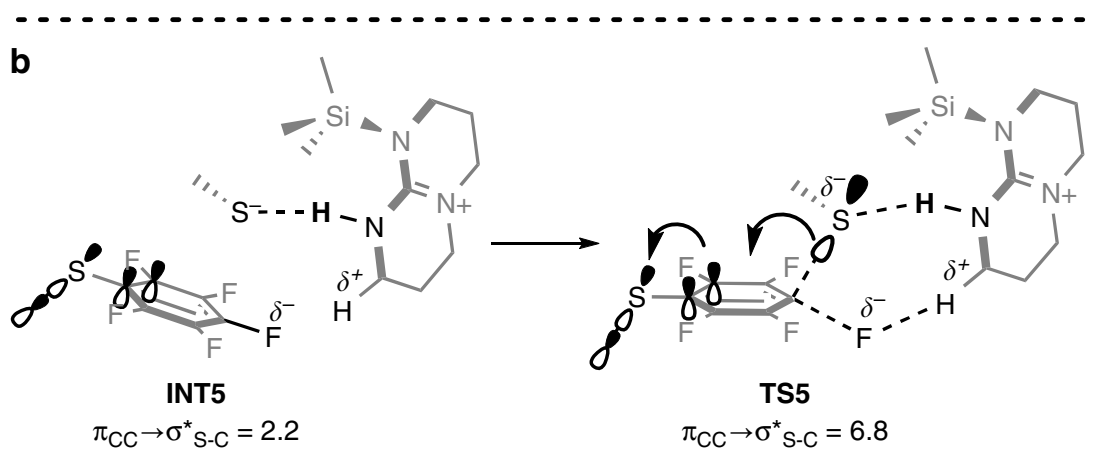

Fig. 3 Computational analysis of transition state stabilization interactions. a NBO analysis of TS2. Highlighted interactions are responsible for stabilization of this TS. Second-order perturbation energies, in $\mathrm{kcal} / \mathrm{mol}$. b NBO analysis of INT5 and TS5. Highlighted interactions are responsible for stabilization of each structure. Second-order perturbation energies, in $\mathrm{kcal} / \mathrm{mol}$

examination of the mechanistic underpinnings of this process reveals a unique, concerted mechanism for the $S_{N} A r$ reaction between silyl protected thioethers and perfluoroarenes, where the organocatalyst plays a critical, dual-activation role. Future work will endeavor to leverage these mechanistic and synthetic insights to further refine control over the polymerization reaction conditions for the development of both new fluorinated materials and 
seamless material processing techniques with minimal byproduct generation.

\section{Methods}

General procedure for polymer synthesis. An $8 \mathrm{ml} \mathrm{screw-cap} \mathrm{vial} \mathrm{equipped} \mathrm{with}$ a magnetic stir-bar was charged with the thioether monomer ( 1 equiv) and fluoroarene (1-1.05 equiv), if solid. Solvent was then added, followed by any monomer that is a liquid (thioether or fluoroarene). The reaction mixture was then stirred to fully mix and dissolve the monomers. The catalyst $(0.5-10 \mathrm{~mol} \%)$ was then added and the reaction mixture was stirred for the indicated time at the specified temperature. Following completion of the reaction, methanol $(8 \mathrm{ml})$ was added to precipitate the polymer. The solid was collected via centrifugation and decanting of the supernatant. Additional methanol $(8 \mathrm{ml})$ was added to the recovered solid and the process centrifugation and decanting was repeated a second time. After drying, the isolated sample was analyzed via NMR and GPC. See Supplementary Figs. for NMR spectra.

Synthesis of $\mathbf{1 b}$. In a nitrogen-filled glovebox and in accordance with the general procedure, a mixture of $1 \mathrm{a}(84 \mu \mathrm{l}, 0.25 \mathrm{mmol})$, hexafluorobenzene $(28 \mu \mathrm{l}, 0.25$ $\mathrm{mmol})$, DBU $(20 \mu \mathrm{l}$ of a $0.062 \mathrm{M}$ stock solution in DMF), and DMF $(0.25 \mathrm{ml})$ were stirred at room temperature for $15 \mathrm{~min}$. After $15 \mathrm{~min}$, the vial was removed from the glovebox and subjected to the workup in accordance with the general procedure, affording the polymer as a white solid. $M_{\mathrm{n}}=33210 \mathrm{~g} / \mathrm{mol}, M_{\mathrm{w}}=120743 \mathrm{~g} / \mathrm{mol}$, $Ð=3.64 .{ }^{1} \mathrm{H}$ NMR $\left(400 \mathrm{MHz}, \mathrm{CDCl}_{3}\right) \delta 2.92(\mathrm{~m}, 4 \mathrm{H}), 1.56(\mathrm{~m}, 4 \mathrm{H}), 1.41$ $(\mathrm{m}, 4 \mathrm{H}) .{ }^{19} \mathrm{~F}$ NMR $\left(128 \mathrm{MHz} \mathrm{CDCl}_{3}\right)-135.07 . T_{\mathrm{g}}$ (DSC): $-18^{\circ} \mathrm{C}$.

Synthesis of $\mathbf{2 b}$. In accordance with the general procedure, a mixture of $\mathbf{1 a}(161 \mu \mathrm{l}$ $0.48 \mathrm{mmol})$, decafluorobiphenyl ( $167 \mathrm{mg}, 0.5 \mathrm{mmol}), \mathrm{DBU}(0.75 \mu \mathrm{l}, 0.005 \mathrm{mmol}$; added as a stock solution in $0.1 \mathrm{ml} D M F)$, and DMF $(0.5 \mathrm{ml})$ were stirred at room temperature for $15 \mathrm{~min}$. Following the workup in accordance with the general procedure, the polymer was isolated as a white solid. $M_{\mathrm{n}}=15701 \mathrm{~g} / \mathrm{mol}, M_{\mathrm{w}}=$ $40353 \mathrm{~g} / \mathrm{mol}, \oplus=2.57 .{ }^{1} \mathrm{H}$ NMR $\left(400 \mathrm{MHz} \mathrm{CDCl}_{3}\right) \delta 3.03(\mathrm{~m}, 4 \mathrm{H}), 1.65(\mathrm{~m}, 4 \mathrm{H})$, $1.48(\mathrm{~m}, 4 \mathrm{H}) .{ }^{19} \mathrm{~F}$ NMR $\left(128 \mathrm{MHz}, \mathrm{CDCl}_{3}\right) \delta-134.6(\mathrm{~m}, 4 \mathrm{~F}),-139.2(\mathrm{~m}, 4 \mathrm{~F})$. $T_{\mathrm{g}}$ (DSC): $16.7^{\circ} \mathrm{C}$. For the analogous TBD catalyzed polymerization the mass recovered was $209 \mathrm{mg}(94 \%)$.

Synthesis of 2c. In a nitrogen filled glovebox and in accordance with the general procedure, a mixture of 2a (197 mg, $0.50 \mathrm{mmol})$, decafluorobiphenyl (168 ml, 0.50 $\mathrm{mmol})$, and DMF $(0.5 \mathrm{mmol})$ were stirred at room temperature for $5 \mathrm{~min}$.

Following the workup in accordance with the general procedure, the polymer was isolated as a white solid (mass recovered: $263 \mathrm{mg}, 94 \%$ ). Note: Polymer retained residual DMF after workup and drying. $M_{\mathrm{n}}=7768 \mathrm{~g} / \mathrm{mol}, M_{\mathrm{w}}=47026 \mathrm{~g} / \mathrm{mol}, \emptyset=$ 6.05. ${ }^{1} \mathrm{H}$ NMR $\left(400 \mathrm{MHz} \mathrm{CDCl}_{3}\right) \delta 7.34(\mathrm{~m}, 8 \mathrm{H}) .{ }^{19} \mathrm{~F} \mathrm{NMR}\left(128 \mathrm{MHz} \mathrm{CDCl}_{3}\right)$ $\delta-132.8(\mathrm{~m}, 4 \mathrm{~F}),-133.0(\mathrm{~m}, 0.5 \mathrm{~F}),-137.9(\mathrm{~m}, 4 \mathrm{~F}),-138.1(\mathrm{~m}, 0.5 \mathrm{~F}) . T_{\mathrm{g}}$ (DSC): $150^{\circ} \mathrm{C}$.

Synthesis of $\mathbf{2 d}$. In a nitrogen-filled glovebox and in accordance with the general procedure, a mixture of $2 \mathrm{a}(197 \mathrm{mg}, 0.50 \mathrm{mmol})$, hexafluorobenzene $(56 \mu \mathrm{l}, 0.50$ $\mathrm{mmol})$, DBU $(20 \mu \mathrm{l}$ of a $0.062 \mathrm{M}$ stock solution in DMF), and DMF $(0.50 \mathrm{ml})$ were stirred at room temperature for $10 \mathrm{~min}$. Following the workup in accordance with the general procedure, the polymer was isolated as a white solid (mass recovered: $218 \mathrm{mg}, 75 \%) . T_{\mathrm{g}}$ (DSC): $48^{\circ} \mathrm{C}$. Note: The low solubility of the resulting polymer prevented full analysis by GPC or NMR.

Synthesis of 2e. In accordance with the general procedure, a mixture of $1 \mathbf{a}(168 \mu \mathrm{l}$, $0.50 \mathrm{mmol})$, bis(4-fluoro-3-nitrophenyl)sulfone (172 $\mathrm{mg}, 0.50 \mathrm{mmol})$, DBU (20 $\mu \mathrm{l}$ of a $0.125 \mathrm{M}$ stock solution in DMF), and DMF $(0.5 \mathrm{ml})$ were stirred at room temperature for the indicated amount of time. Following the workup in accordance with the general procedure, the polymer was isolated as a light yellow solid (mass recovered: $145 \mathrm{mg}, 64 \%) . T_{\mathrm{g}}(\mathrm{DSC}): 28^{\circ} \mathrm{C}$. Note: The low solubility of the resulting polymer prevented full analysis by GPC or NMR.

Data availability. All the data are available from authors upon reasonable request.

Received: 6 February 2017 Accepted: 8 June 2017

Published online: 01 August 2017

\section{References}

1. Sanji, T. \& Iyoda, T. Transition-metal-free controlled polymerization of 2perfluoroaryl-5-trimethylsilylthiophenes. J. Am. Chem. Soc. 136, 10238-10241 (2014).

2. Dutta, T., Woody, K. B., Parkin, S. R., Watson, M. D. \& Gierschner, J. Conjugated polymers with large effective stokes shift: benzobisdioxole-based poly(phenylene ethynylene)s. J. Am. Chem. Soc. 131, 17321-17327 (2009).
3. Dutta, T., Woody, K. B. \& Watson, M. D. Transition-metal-free synthesis of poly(phenylene ethynylene)s with alternating aryl-perfluoroaryl units. J. Am. Chem. Soc. 130, 452-453 (2008).

4. Woody, K. B., Bullock, J. E., Parkin, S. R. \& Watson, M. D. Alternating arene-perfluoroarene poly(phenylene ethynylenes). Macromolecules 40, 4470-4473 (2007).

5. Wang, Y. \& Watson, M. D. Transition-metal-free synthesis of alternating thiophene-perfluoroarene copolymers. J. Am. Chem. Soc. 128, 2536-2537 (2006).

6. Tian, S. et al. Postpolymerization of a fluorinated and reactive poly(aryl ether): an efficient way to balance the solubility and solvent resistance of the polymer. ACS Appl. Mater. Interfaces 6, 20437-20443 (2014).

7. Calzolari, A., Vercelli, B., Ruini, A., Virgili, T. \& Pasini, M. Fluorine-induced enhancement of the oxidation stability and deep-blue optical activity in conductive polyfluorene derivatives. J. Phys. Chem. C 117, 26760-26767 (2013).

8. Aljoumaa, K., Qi, Y., Ding, J. \& Delaire, J. A. Synthesis and characterizations of highly fluorinated poly(arylene ether)s for quadratic nonlinear optics. Macromolecules 42, 9275-9288 (2009).

9. Ding, J. \& Qi, Y. The preparation and characterization of highly fluorinated poly(arylene alkylene ether)s. Macromolecules 41, 751-757 (2008).

10. Kim, J.-P. et al. Fluorinated poly(arylene ether sulfide) for polymeric optical waveguide devices. Macromolecules 34, 7817-7821 (2001)

11. Miyatake, K., Yamamoto, K., Yokoi, Y. \& Tsuchida, E. Novel synthesis of linear perfluorinated poly(phenylene sulfide) from aryl sulfoxide. Macromolecules $\mathbf{3 1}$ 403-407 (1998).

12. Goodwin, A. A., Mercer, F. W. \& McKenzie, M. T. Thermal behavior of fluorinated aromatic polyethers and poly(ether ketone)s. Macromolecules 30, 2767-2774 (1997).

13. Pace, C. J. \& Gao, J. Exploring and exploiting polar- $\pi$ interactions with fluorinated aromatic amino acids. Acc. Chem. Res. 46, 907-915 (2013).

14. Williams, J. H., Cockcroft, J. K. \& Fitch, A. N. Structure of the lowest temperature phase of the solid benzene-hexafluorobenzene adduct. Angew. Chem. Int. Ed. 31, 1655-1657 (1992)

15. Vo, C. D., Kilcher, G. \& Tirelli, N. Polymers and sulfur: what are organic polysulfides good for? Preparative strategies and biological applications. Macrom. Rapid Commun. 30, 299-315 (2009).

16. Liu, J., Chen, G. \& Fang, X. Synthesis and characterization of high performance poly(thioether imide)s via aromatic nucleophilic substitution reaction of isomeric AB-type monomers. Polym. Bull. 72, 3269-3282 (2015).

17. Nakagawa, Y., Suzuki, Y., Higashihara, T., Ando, S. \& Ueda, M. Synthesis of highly refractive poly(phenylene thioether)s containing a binaphthyl or diphenylfluorene unit. Polym. Chem. 3, 2531-2536 (2012).

18. Nakagawa, Y., Suzuki, Y., Higashihara, T., Ando, S. \& Ueda, M. Synthesis of highly refractive poly(phenylene thioether) derived from 2,4-dichloro-6alkylthio-1,3,5-triazines and aromatic dithiols. Macromolecules 44, 9180-9186 (2011).

19. Seesukphronrarak, S., Kawasaki, S., Kobori, K. \& Takata, T. Synthesis of fluorene-based high performance polymers. I. Poly(arylene thioether)s with excellent solubility and high refractive index. J. Polym. Sci. A Polym. Chem. 45, 3073-3082 (2007).

20. In, I. \& Kim, S. Y. Poly(arylene thioether) synthesis via nitro-displacement reaction. J. Polym. Sci. A Polym. Chem. 44, 2440-2447 (2006).

21. In, I. \& Kim, S. Y. Synthesis of poly(arylene thioether)s from protected dithiols and aromatic difluorides with an organic base. J. Polym. Sci. A Polym. Chem. 43 2021-2027 (2005).

22. Masaki, S., Sato, N., Nishichi, A., Yamazaki, S. \& Kimura, K. Fluorinated poly (aryl thioether)s and poly(aryl sulfone)s derived from 2,3,4,5,6pentafluorobenzoic acid. J. Appl. Polym. Sci. 108, 498-503 (2008).

23. Zhao, Z., Ma, X., Zhang, A. \& Song, N. A unique stepped multifunctionality of perfluorinated aryl compound and its versatile use in synthesizing grafted polymers with controlled structures and topologies. J. Polym. Sci. A Polym. Chem. 49, 2423-2433 (2011).

24. Gierczyk, B. \& Schroeder, G. Synthesis of supramolecular cyclosiloxane ligands. Mendeleev Commun. 19, 75-77 (2009).

25. Lowe, J. N. et al. Polyvalent interactions in unnatural recognition processes. J. Org. Chem. 69, 4390-4402 (2004).

26. Langner, C., Meier-Haack, J., Voit, B. \& Komber, H. The stepped reaction of decafluorobiphenyl with thiophenol studied by in situ ${ }^{19}$ F NMR spectroscopy. J. Fluorine Chem. 156, 314-321 (2013).

27. García, J. M. et al. Meisenheimer Complex inspired catalyst- and solvent-free synthesis of noncyclic poly(aryl ether sulfone)s. Macromolecules 47, 8131-8136 (2014).

28. Kricheldorf, H. R. \& Bier, G. New polymer syntheses: II. preparation of aromatic poly(ether ketone)s from silylated bisphenols. Polymer 25, 1151-1156 (1984).

29. Dong, J., Sharpless, K. B., Kwisnek, L., Oakdale, J. S. \& Fokin, V. V. SuFExbased synthesis of polysulfates. Angew. Chem. Int. Ed. 53, 9466-9470 (2014). 
30. Birchall, J. M., Green, M., Haszeldine, R. N. \& Pitts, A. D. The mechanism of the nucleophilic substitution reactions of polyfluoroarenes. Chem. Commun. 338-339 (1967).

31. Brooke, G. M. The preparation and properties of polyfluoro aromatic and heteroaromatic compounds. J. Fluorine Chem. 86, 1-76 (1997).

32. Gold, B., Shevchenko, N., Bonus, N., Dudley, G. B. \& Alabugin, I. V. Selective transition state stabilization via hyperconjugative and conjugative assistance: stereoelectronic concept for copper-free click chemistry. J. Org. Chem. 77, 75-89 (2012).

33. Vatsadze, S. Z., Loginova, Y. D., Gomes, G. \& Alabugin, I. V. Stereoelectronic chameleons: the donor-acceptor dichotomy of functional groups. Chem. Eur. J. 23, 3225-3245 (2016).

34. Peterson, P. W., Shevchenko, N. \& Alabugin, I. V. "Stereoelectronic umpolung": converting a p-donor into a $\sigma$-acceptor via electron injection and a conformational change. Org. Lett. 15, 2238-2241 (2013).

\section{Acknowledgements}

G.G. is grateful to IBM for the 2016 IBM PhD Scholarship. We thank Andy T. Tek (IBM) for conducting thermal analyses.

\section{Author contributions}

J.L.H. and I.V.A.: Oversaw the project. N.H.P.: Synthesized all monomers and performed all polymerization experiments. G.d.P.G. and G.O.J.: Performed the computational investigations. M.F.: Prepared braids for DMA of polymers. All authors contributed to paper writing.

\section{Additional information}

Supplementary Information accompanies this paper at doi:10.1038/s41467-017-00186-3.

Competing interests: The authors declare no competing financial interests.

Reprints and permission information is available online at http://npg.nature.com/ reprintsandpermissions/

Change History: A correction to this article has been published and is linked from the HTML version of this article.

Publisher's note: Springer Nature remains neutral with regard to jurisdictional claims in published maps and institutional affiliations.

(c) (i) Open Access This article is licensed under a Creative Commons Attribution 4.0 International License, which permits use, sharing, adaptation, distribution and reproduction in any medium or format, as long as you give appropriate credit to the original author(s) and the source, provide a link to the Creative Commons license, and indicate if changes were made. The images or other third party material in this article are included in the article's Creative Commons license, unless indicated otherwise in a credit line to the material. If material is not included in the article's Creative Commons license and your intended use is not permitted by statutory regulation or exceeds the permitted use, you will need to obtain permission directly from the copyright holder. To view a copy of this license, visit http://creativecommons.org/ licenses/by/4.0/.

(C) The Author(s) 2017 